\title{
Um Desenvolvimento Numérico nas Equações Dinâmicas de Sólitons em Fibras Óticas
}

\author{
A Numerical Development in the Dynamical Equations of \\ Solitons in Optical Fibers
}

\author{
Diogo Albino de Queiroz ${ }^{1}$; Paulo Laerte Natti²; Neyva Maria Lopes Romeiro ${ }^{3}$; \\ Érica Regina Takano Natti ${ }^{4}$
}

\section{Resumo}

Avaliamos a resolução numérica de um sistema de equações diferenciais não-lineares, que descreve a propagação de sólitons em fibras óticas dielétricas, por meio do método de elementos finitos, implementado a partir de formulações streamline upwind Petrov-Galerkin (SUPG) e consistent approximate upwind (CAU).

Palavras-chave: Fibra ótica. Sóliton. Método de elementos finitos.

\begin{abstract}
It was evaluated the numerical resolution of a nonlinear differential equations system that describes the solitons propagation in dielectric optical fibers, through the method of finite elements, which is implemented based on Streamline Upwind Petrov-Galerkin (SUPG) and Consistent Approximate Upwind (CAU) formulations.
\end{abstract}

Key words: Optical fiber. Soliton. Finite elements method.

\footnotetext{
${ }^{1}$ Bolsista de Iniciação Científica IC/UEL. Aluno de graduação em Ciência da Computação do Departamento de Computação da Universidade Estadual de Londrina, Londrina- PR. E-mail: daqueiroz@ dc.uel.br.

${ }^{2}$ Professor Doutor do Departamento de Matemática da Universidade Estadual de Londrina, Londrina - PR. Email: plnatti@ uel.br.

${ }^{3}$ Professora Doutora do Departamento de Matemática da Universidade Estadual de Londrina, Londrina - PR. E-mail: nromeiro@uel.br.

${ }^{4}$ Professora Doutora da Pontifícia Universidade Católica do Paraná, Londrina -PR. E-mail: erica.takano@uol.com.br.
} 


\section{Introdução}

Recentemente, considerável atenção tem sido dada ao estudo de uma classe de equações diferenciais de campos acoplados com nãolinearidades de segunda ordem nos campos. Uma grande variedade de fenômenos pode ser descrita por esses sistemas de equações, em particular, fenômenos não-lineares em ótica. Comunicação ótica (HAUS; WONG, 1996), light bullets (MALOMED et al., 2005), aparelhos óticos tais como interferômetros do tipo Mach-Zehnder (ASSANTO et al., 1993), couplers direcionais (LEDERER et al., 1990) e outros (TAYLOR, 1992; HASEGAWA; KODAMA, 1995) são descritos por tais equações.

Nosso interesse em estudar esses sistemas de equações é aplicá-las ao estudo da estabilidade de sinais em fibras óticas com geometria transversal retangular, nas quais as ondas estacionárias do tipo sólitons são possíveis (GALLEAS et al., 2003; YMAI et al., 2004).

Os sólitons podem ser utilizados em comunicações a longa distância sem a necessidade de estações repetidoras, inclusive em comunicações transoceânicas, como foi proposto por Hasegawa (1984). A comunicação convencional utiliza sinais lineares, no formato NRZ (nonreturn to zero), que são regenerados eletronicamente, e, então, retransmitidos. A localização e a distância das estações repetidoras são escolhidas, de modo que ainda seja possível recuperar o sinal com uma taxa de erro menor que 1 bit em $10^{9}$ bits. Atualmente, essa distância varia entre 120-150 Km. Por outro lado, Nagazawa et al. (1991) haviam propagado sólitons em laboratório, num sistema fechado (em loop) de fibras, a distâncias superiores a $10^{6} \mathrm{Km}$ já em 1991, utilizando moduladores de amplitude.

As propriedades dielétricas que fibras óticas devem apresentar, de modo que a comunicação via sólitons seja mais estável e eficaz que a comunicação via sinais lineares no formato NRZ, é de grande interesse comercial e tecnológico. Por apresentar diversas aplicações potenciais em Telecomunicações, estudos sobre a propagação e a estabilidade de sólitons têm tido um grande crescimento nos últimos quinze anos (HAUS; WONG, 1996; QIN; DAI; ZHANG, 2005).

Neste trabalho faremos um estudo numérico de sistemas de equações diferenciais, assintoticamente equivalentes à equação de Schrödinger não-linear (ZAKHAROV; SABAT, 1972; HASEGAWA; TAPPERT, 1973; MENYUK; SCHIEK; TORNER, 1994). Essas equações descrevem a evolução de ondas tipo sólitons em fibras óticas com nãolinearidades quadráticas, também chamadas de guias dielétricos do tipo $\chi^{(2)}$. Este estudo será implementado para um sistema simplificado de equações diferenciais parciais (EDP's), o qual apresenta soluções analíticas conhecidas (GALLEAS et al., 2003). Desse modo iremos ajustar e avaliar um procedimento numérico desenvolvido por Romeiro e colaboradores, baseado no método de elementos finitos com formulações do tipo Streamline Upwind Petrov-Galerkin - SUPG (GALEÃO; CARMO, 1988; BROOKS; HUGHES, 1982; HUGHES; FRANCA; MALLET, 1986) e do tipo Consistent Approximate Upwind - CAU (ALMEIDA; GALEÃO, 1993; GALEÃO et al., 2004).

A partir deste trabalho, será possível estudar a solução numérica de sistemas de EDP's mais complexos, que descrevem de forma realista a propagação de sólitons em materiais dielétricos. Esses sistemas, ditos realistas, não têm soluções analíticas conhecidas, de forma que o tratamento numérico é uma maneira possível para simular e otimizar a propagação destas ondas em função das propriedades dielétricas dos materiais (YMAI et al., 2004).

Para o desenvolvimento deste trabalho propomos três etapas. Na primeira etapa, a partir de um sistema de EDP's, que descreve a propagação de sóliton ideais em fibras óticas do tipo $\chi^{(2)}$, deduzimos um sistema de equações diferenciais ordinárias (EDO's) equivalente. Na segunda etapa, apresentamos as diretrizes, referentes ao procedimento numérico, para a resolução do sistema de EDO's obtido. Na terceira etapa, comparando graficamente as soluções analíticas conhecidas com as soluções numéricas obtidas, analisamos o método numérico quando 
aplicado ao sistema de equações diferenciais discutidos neste trabalho.

\section{Dinâmica Longitudinal de Campos Acoplados em Dielétricos $\chi^{(2)}$}

O sistema de EDP's não-lineares, obtido a partir das equações de Maxwell, descreve a evolução longitudinal de duas ondas eletromagnéticas acopladas (modos fundamental e segundo harmônico) em guias de ondas (fibras óticas) com nãolinearidades do tipo $\chi^{(2)}$. Esse sistema é apresentado nas equações (1) e (2) (GALLEAS et al., 2003).

$$
\begin{aligned}
& i \frac{\partial a_{1}}{\partial \xi}-\frac{r}{2} \frac{\partial^{2} a_{1}}{\partial s^{2}}+a_{1}^{*} a_{2} \exp (-i \beta \xi)=0 \\
& i \frac{\partial a_{2}}{\partial \xi}-i \delta \frac{\partial a_{2}}{\partial s}-\frac{\alpha}{2} \frac{\partial^{2} a_{2}}{\partial s^{2}}+a_{1}^{2} \exp (i \beta \xi)=0
\end{aligned}
$$

Onde $a_{1}(s, \xi)$ e $a_{2}(s, \xi)$ são variáveis complexas e representam as amplitudes normalizadas das ondas fundamental e segundo harmônico, respectivamente.

O sistema dado em (1) e (2) apresenta soluções analíticas do tipo sólitons para certas faixas de valores de $r, \alpha, \beta$ e $\delta$ (YMAI et al., 2004). Vejamo como estas quantidades caracterizam as propriedades nãolineares (dielétricas) da fibra ótica. No limite $|\beta| \rightarrow \infty$, as equações diferenciais (1) e (2) reduzem-se à equação de Schrödinger não-linear (NLSE) (MENYUK; SCHIEK; TORNER, 1994), correspondendo ao limite de incompatibilidade de fase (ondas fora de fase). Consequentemente, a quantidade $\beta$ mede a taxa de geração do segundo harmônico, ou ainda, a intensidade de não-linearidade do material. A quantidade $\alpha$ mede a dispersão relativa das velocidades de grupo das ondas fundamental e segundo harmônico no material (fibra ótica). Para valores de $|\alpha|>1$, a onda do segundo harmônico possui dispersão maior que a onda fundamental e para valores de $|\alpha|<1$ é a onda fundamental que tem dispersão maior. A quantidade $r$ é um indicador do regime de dispersão da onda fundamental. Quando temos $r=+1$, a onda fundamental encontra-se no regime de dispersão dito normal, porém se $r=-1$, a onda fundamental encontra-se no regime de dispersão dito anômalo. Enfim, a quantidade $\delta$ mede a diferença das velocidades de grupo dos modos fundamental e segundo harmônico, e assume valores não-nulos, por exemplo, em meios anisotrópicos, quando os vetores de Poynting destes modos estão desalinhados (walkoff wave). No trabalho de Galleas et al. (2003), encontra-se uma descrição pormenorizada da dedução da solução das equações (1) e (2) e da interpretação das quantidades dielétricas $\alpha, \beta, \delta$ e $r$, relacionandoas com as propriedades óticas da fibra.

Observamos que podemos escolher as características da onda sóliton a ser propagada na fibra ótica (velocidade, largura, amplitude, estabilidade, etc..), selecionando ou propondo materiais com as propriedades dielétricas $\alpha, \beta, \delta$ e $r$ adequadas.

As soluções analíticas tipo sólitons das equações (1) e (2) são conhecidas (MENYUK; SCHIEK; TORNER, 1994; GALLEAS et al., 2004) e apresentadas em (3) e (4),

$$
\begin{aligned}
& a_{1}(s, \xi)=|\beta|^{1 / 2} \hat{a}_{1}(s, \xi) \\
& a_{2}(s, \xi)=\hat{a}_{2}(s, \xi) \exp (i \beta \xi)
\end{aligned}
$$

onde

$$
\hat{a}_{1}= \pm \frac{3}{2(\alpha-2 r)} \sqrt{\frac{\alpha r}{|\beta|}}\left(\frac{\delta^{2}}{2 \alpha-r}+\beta\right) \times
$$

$$
\begin{aligned}
& \operatorname{sch}^{2}\left[ \pm \sqrt{\frac{1}{2(2 r-\alpha)}\left(\frac{\delta^{2}}{2 \alpha-r}+\beta\right)}\left(s-\frac{r \delta}{2 \alpha-r} \xi\right)\right] \times \\
& \exp \left\{i\left[\frac{r \delta^{2}(4 r-5 \alpha)}{2(2 \alpha-r)^{2}(2 r-\alpha)}-\frac{r \beta}{2 r-\alpha}\right] \xi-\frac{i \delta}{2 \alpha-r} s\right\}
\end{aligned}
$$


$\hat{a}_{2}=\frac{3 r}{2(\alpha-2 r)}\left[\frac{\delta^{2}}{2 \alpha-r}+\beta\right] \times$

$\operatorname{sch}^{2}\left[ \pm \sqrt{\frac{1}{2(2 r-\alpha)}\left(\frac{\delta^{2}}{2 \alpha-r}+\beta\right)}\left(s-\frac{r \delta}{2 \alpha-r} \xi\right)\right] \times$

$\exp \left\{2 i\left[\frac{r \delta^{2}(4 r-5 \alpha)}{2(2 \alpha-r)^{2}(2 r-\alpha)}-\frac{r \beta}{2 r-\alpha}\right] \xi-\frac{2 i \delta}{2 \alpha-r} s\right\}$

Por simplicidade, consideraremos $\delta=0$ no desenvolvimento numérico de (1) e (2). No caso da propagação de sólitons em fibras óticas ordinárias, e em situações não-críticas, o fenômeno walk-off wave pode ser desconsiderado (ARTIGAS; TORNER; AKHMEDIEV, 1999), o que justifica tomar $\delta=0$ nestas situações. Assim, o sistema de EDP's torna-se separável e as soluções estacionárias de (1) e (2) têm a forma

$a_{v}=(-1)^{v} U_{v}(s) \exp \left(i \kappa_{v} \xi\right)$

onde $v=1,2$. O cálculo das derivadas parciais é dado por

$\frac{\partial a_{v}}{\partial \xi}=(-1)^{v} i \kappa_{v} U_{v}(s) \exp \left(i \kappa_{v} \xi\right)$

$\frac{\partial a_{v}}{\partial s}=(-1)^{v} \exp \left(i \kappa_{v} \xi\right) \frac{\partial U_{v}}{\partial s}$

$\frac{\partial^{2} a_{v}}{\partial s^{2}}=(-1)^{v} \exp \left(i \kappa_{v} \xi\right) \frac{\partial^{2} U_{v}}{\partial s^{2}}$

Cabe observar que $a_{1}^{*}=-U_{1}(s) \exp \left(-i \kappa_{1} \xi\right)$. Substituindo (7) e (10) em (1), obtém-se:

$\kappa_{1} U_{1} \exp \left(i \kappa_{1} \xi\right)+\frac{r}{2} \exp \left(i \kappa_{1} \xi\right) \frac{\partial^{2} U_{1}}{\partial s^{2}}-$

$U_{1} U_{2} \exp \left[i\left(-\kappa_{1}+\kappa_{2}-\beta\right) \xi\right]=0$

Analogamente, substituindo (7) e (10) em (2), segue que

$\kappa_{2} U_{2} \exp \left(i \kappa_{2} \xi\right)+\frac{\alpha}{2} \exp \left(i \kappa_{2} \xi\right) \frac{\partial^{2} U_{2}}{\partial s^{2}}-$

$U_{1}^{2} \exp \left[i\left(2 \kappa_{1}+\beta\right) \xi\right]=0$
(6) Tomando $\kappa_{2}=2 \kappa_{1}+\beta$, condição necessária para que (1) e (2) tenham soluções do tipo (7), obtemos o sistema de EDO's que descreve a evolução de $U_{1}(s)$ e $U_{2}(s)$, ou seja,

$$
\begin{aligned}
& \frac{r}{2} \frac{\partial^{2} U_{1}}{\partial s^{2}}+\kappa_{1} U_{1}-U_{1} U_{2}=0 \\
& \frac{\alpha}{2} \frac{\partial^{2} U_{2}}{\partial s^{2}}+\kappa_{2} U_{2}-U_{1}^{2}=0
\end{aligned}
$$

Onde todas as variáveis e constantes são reais. As constantes $r, \alpha$ e $\beta$ são obtidas a partir das propriedades materiais da fibra ótica, enquanto o chamado deslocamento não-linear do número de onda $\kappa_{v}$, para $v=1,2$, parametriza uma família de soluções. Na próxima etapa, iremos descrever o procedimento numérico utilizado nas equações (13) e (14).

\section{Procedimento Numérico}

Para resolver numericamente o sistema de equações diferenciais dado em (13) e (14), utilizamos o método de elementos finitos obtido a partir da formulação de Petrov-Galerkin (GALEÃO; CARMO, 1988). Sabe-se que simulações numéricas calculadas por elementos finitos usuais, tais como o método de Galerkin, apresentam dificuldades numéricas relacionadas à deficiência na estabilidade, gerando assim oscilações espúrias (ALMEIDA, et al., 2004). Para contornar este problema, são usados métodos estabilizados do tipo Streamline Upwind Petrov-Galerkin - SUPG (GALEÃO; CARMO, 1988; BROOKS; HUGHES, 1982; HUGHES; FRANCA; MALLET, 1986) e do tipo Consistent Approximate Upwind - CAU (ALMEIDA; GALEÃO, 1993; GALEÃO et al., 2004).

Um código computacional em FORTRAN 90, desenvolvido por um dos autores (ROMEIRO; CASTRO; LANDAU, 2003), foi ajustado para simular nosso problema. Na etapa seguinte, comparando graficamente as soluções numéricas obtidas com as soluções analíticas conhecidas, poderemos tirar conclusões sobre a metodologia numérica empregada. 


\section{Resultados Numéricos e Analíticos}

Para o código que calcula a solução numérica das equações (13) e (14), são fornecidos valores para as quantidades dielétricas $\alpha, \beta \mathrm{e} r$, que definem as características do sóliton a ser propagado na fibra ótica. Experimentalmente, para fibras óticas comerciais, estas quantidades podem assumir os seguintes valores: $\alpha=-1 / 2, \beta=-1 / 2$ e $r=-1$. É importante observar que, nas equações (13) e (14), todas as variáveis e quantidades dielétricas são adimensionais (GALLEAS et al., 2003). A variável temporal $\xi$ é fixada em $\xi=10$, de modo que a variável espacial $s$ descreverá a forma do envelope da onda sóliton, ao longo da fibra ótica, para este "instante" fixado. Resta ainda fornecer as condições iniciais, ou ainda, o formato inicial do envelope da onda que será injetado na fibra ótica. Novamente, com o objetivo de simplificar os procedimentos, quando da comparação das soluções numérica e analítica, tomamos como condição inicial no contorno esquerdo da fibra ótica, uma onda tipo sóliton. Tomando $s=0$ nas soluções analíticas (5) e (6), temse de (7) que as condições no contorno esquerdo são $U_{1}(0, \xi)=\frac{-3 \beta \sqrt{\alpha r}}{2(\alpha-2 r)} \mathrm{e} U_{2}(0, \xi)=\frac{3 \beta r}{2(\alpha-2 r)}$

Para o contorno direito, o gradiente de ambas as ondas são fixados em zero, pois para distâncias $s$ suficientemente grandes as amplitudes $U_{1}$ e $U_{2}$ tendem a zero, de modo que a taxa de variação (gradiente) destas no contorno direito também tendem a zero.

Das equações (13) e (14), considerando uma partição com 30 elementos, consequentemente 31 nós, e com intervalos $\Delta s=0.5$, obtivemos os resultados numéricos que podem ser observados na figura 1. Como esperado, as condições no contorno esquerdo, no instante $\xi=10$, fazem com que a onda sóliton esteja centrada em $s=0$ e tenda a zero para valores maiores da variável $s$.

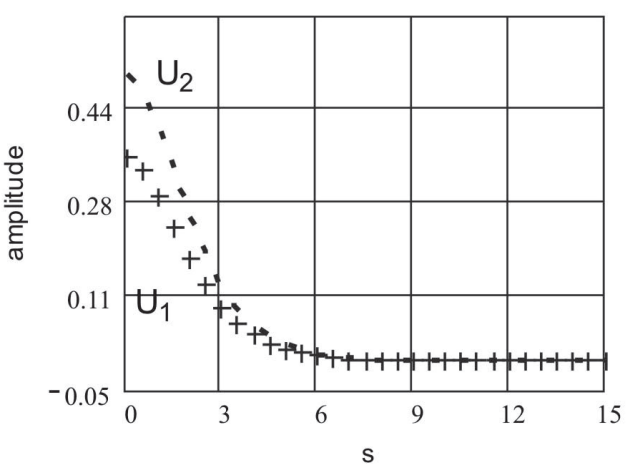

Figura 1. Resultados numéricos, em $\xi=10$, de $U_{1}(s, \xi)$ (símbolo) e $U_{2}(s, \xi)$ (linha tracejada).

Substituindo em (7) as soluções numéricas obtidas para $U_{1}(s, \xi)$ e $U_{2}(s, \xi)$, calculamos as amplitudes complexas $a_{1}(s, 10)$ e $a_{2}(s, 10)$ das equações (1) e (2). Na figura 2, apresentamos as partes real e imaginária dessas amplitudes.

Enfim, as soluções numéricas apresentadas na figura 2 são graficamente comparadas com as soluções analíticas, conhecidas na literatura (YMAI

(a) Soluções Reais

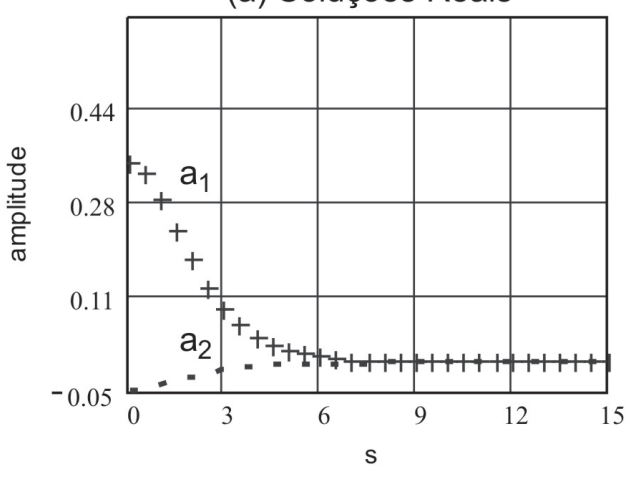

(b) Soluções Imaginárias

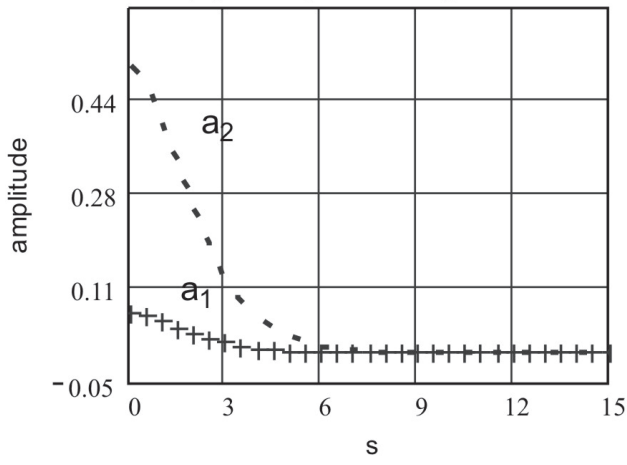

Figura 2. Soluções numéricas, em $\xi=10$, das amplitudes $a_{1}(s, \xi)$ (símbolo) e $a_{2}(s, \xi)$ (linha tracejada). (a) Parte real das soluções, e (b) parte imaginária das soluções. 
et al., 2004), apresentadas em (5) e (6). Para realizar a comparação de forma consistente, entre as soluções numéricas e exatas, tomamos em $\xi=10$ os valores $\alpha=-1 / 2, \beta=-1 / 2, r=-1$ e $\delta=0$ para as quantidades dielétricas em (5) e (6). Os resultados podem ser observados nas figuras 3 e 4 .

(a) Soluções de $a_{1}$

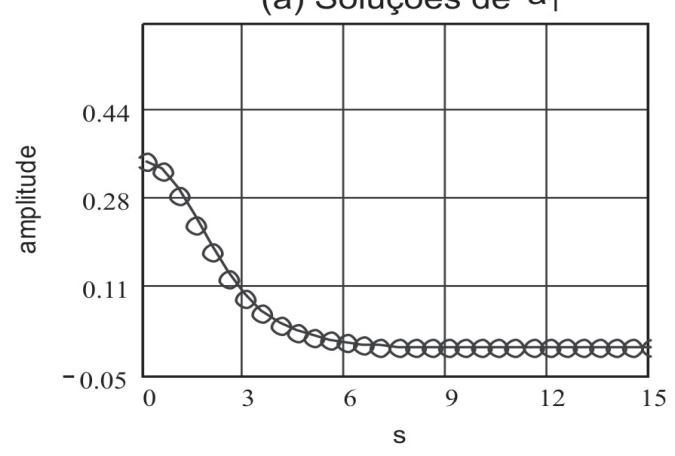

(b) Soluções de $a_{2}$

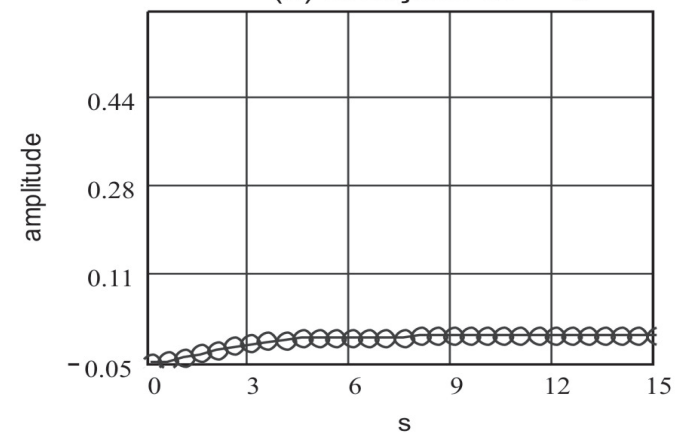

Figura 3. Comparações entre as soluções numéricas (símbolo) e analíticas (linha contínua). (a) Solução $\operatorname{Re}\left(a_{1}\right)$, e (b) solução $\operatorname{Re}\left(a_{2}\right)$.

(a) Soluções de $a_{1}$

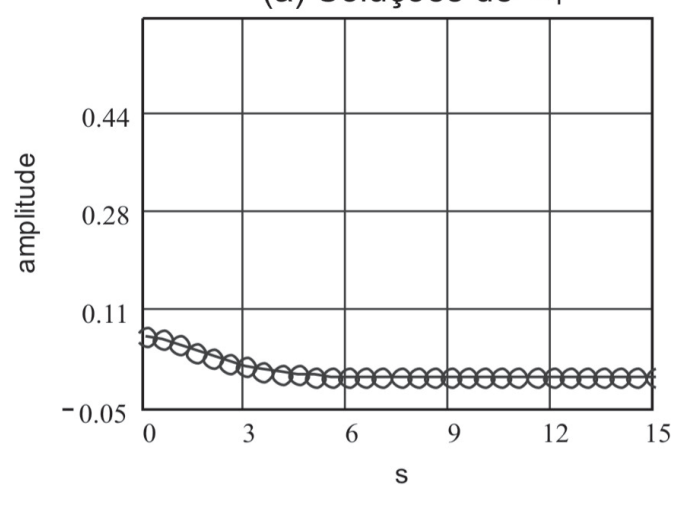

(b) Soluções de $a_{2}$

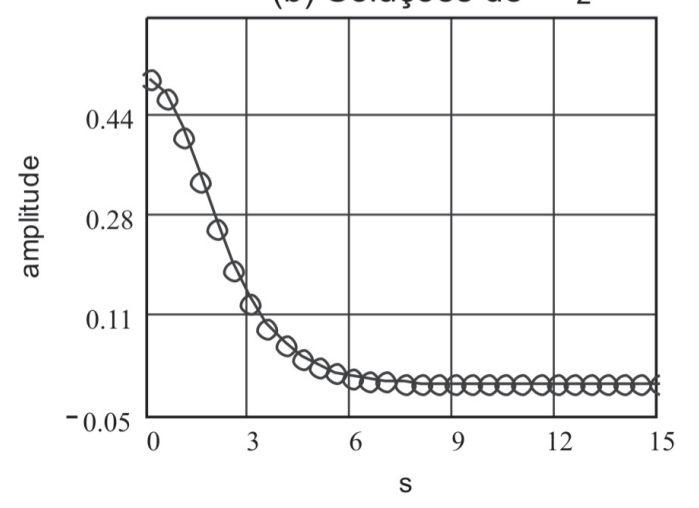

Figura 4. Comparações entre as soluções numéricas (símbolo) e analíticas (linha contínua). (a) Solução $\operatorname{Im}\left(a_{1}\right)$, e (b) solução $\operatorname{Im}\left(a_{2}\right)$.

\section{Conclusões}

Verificamos que o sistema de equações diferenciais não-lineares, que descreve a evolução longitudinal de sólitons (duas ondas eletromagnéticas acopladas - modos fundamental $a_{1}$ e segundo harmônico $a_{2}$ ) em fibras óticas do tipo $\chi^{(2)}$, tornase um sistema de EDP's separável, quando $\delta=0$, cujas soluções estacionárias podem ser obtidas numericamente, veja as figuras 1 e 2 , segundo o desenvolvimento numérico descrito anteriormente.

Além disto, a partir dos resultados apresentados nas figuras 3 e 4 , observamos que as soluções analíticas descritas em (3) e (6), considerando $\delta=0$, comparadas com as soluções numéricas de (13) e (14), substituídas em (7), são graficamente muito próximas. Desses resultados podemos concluir que o método numérico permitiu obter soluções aceitáveis.

Como perspectiva de continuidade deste trabalho, aplicaremos o método numérico desenvolvido na simulação da propagação de sinais do tipo sólitons ideais, ao caso de fibras óticas não-ideais, onde estudaremos como pequenas perturbações locais afetam a estabilidade destes. Sabe-se que inomogeneidades (difusão de moléculas hidrogênio, bolhas, impurezas metálicas,...) e defeitos (variação do diâmetro, rugosidade, sinuosidade no eixo, microcurvaturas, emendas, ..) da fibra ótica são as 
principais causas de absorção-dissipação da onda sóliton (RAGHAVAN; AGRAWAL, 2000; STROBEL, 2004). Supondo uma absorção da ordem de $2 \mathrm{~dB} / \mathrm{Km}$, e um processo de bombeamento do sinal com geração de ruído, estudaremos numericamente a estabilidade da propagação de sólitons por grandes distâncias (WERNER; DRUMMOND, 1993). Nestas modelagens não-ideais a descrição analítica da propagação do sinal não é conhecida e somente um tratamento numérico poderá indicar novos caminhos para as Ciências dos Materiais Dielétricos e das Telecomunicações.

\section{Agradecimentos}

Os autores gostariam de agradecer à Universidade Estadual de Londrina pelo apoio financeiro a pesquisa, através do Programa FAEPE/2005 - Fundo de Apoio ao Ensino, Pesquisa e Extensão, e através da bolsa de Iniciação Científica, Programa PROIC/UEL, fornecida a D. A. de Queiroz.

\section{Referências}

AlmeidA, R. C. C.; GAlEÃO, A. C. The generalized CAU operator for the compressible Euler and Navier-Stokes equations. In: INTERNATIONAL CONFERENCE ON NUMERICAL METHODS IN LAMINAR AND TURBULENT FLOWS, 8., Swansea, 1993. Proceedings... Swansea: C. Taylor, 1993.

ALMEIDA, R. C.; GALEAO, A. C.; MALTA, S. M. C.; LOULA, A. F. D. Finite element analysis of convection dominated reaction-diffusion problems. Applied Numerical Mathematics, Amsterdam, v.48, n.2, p.205-222, feb. 2004.

ARTIGAS, D.; TORNER, L.; AKHMEDIEV, N. N. Dynamics of quadratic soliton excitation. Optics Communications, Amsterdam, v.162, n.4/6, p.347356, apr. 1999.
ASSANTO, G.; STEGEMAN, G.; SHEIK-BAHAE, M.; STRYLAND, E. V. All optical-switching devices based on large nonlinear phase shifts from second harmonic generation. Applied Physics Letters, New York, v.62, n.12, p.1323-1325, mar. 1993.

BROOKS, A. N.; HUGHES, T. J. R. Streamline upwind Petrov-Galerkin formulation for convectiondominated flows with particular emphasis on the incompressible Navier-Stokes equations. Computer Methods in Applied Mechanics and Engineering, Amsterdam, v.32, n.1/3, p.199-259, 1982.

GALEÃO, A. C.; ALMEIDA, R. C.; MALTA, S. M. C.; LOULA, A. F. D. Finite element analysis of convection dominated reaction-diffusion problems. Applied Numerical Mathematics, Amsterdam, v.48, n.2, p.205-222, feb. 2004.

GALEÃO, A. C.; CARMO, E. G. D. A consistent approximate upwind Petrov-Galerkin method for convection-dominated problems. Computer Methods in Applied Mechanics Engineering, Amsterdam, v.68, n.1, p.83-95, may 1988.

GALLEAS, W.; YMAI, L. H.; NATTI, P. L.; TAKANO NATTI, E. R. Ondas do tipo sólitons em dielétricos. Revista Brasileira de Ensino de Física, São Paulo, v.25, n.3, p.294-304, 2003.

HASEGAWA, A. Numerical study of optical soliton transmission periodically by the stimulated Raman process. Applied Optics, New York, v.23, n.19, p.3302-3309, 1984.

HASEGAWA, A.; KODAMA Y. Solitons in optical communications. Oxford: Claredon, 1995.

HASEGAWA, A.; TAPPERT, F. Transmission of stationary nonlinear optical pulses in dispersive dielectric fibers. I. Anomalous dispersion. Applied Physics Letters, New York, v.23, n.4, p.172-172, 1973. 
HAUS, H. A; WONG, W. S. Soliton in optical communications. Reviews of Modern Physics, Minneapolis, v.68, n.2, p.423-444, apr. 1996.

HUGHES, T. J. R.; FRANCA, L. P.; MALLET, M. A new finite element formulation for computational fluid dynamics: I. Symmetric forms of the compressible Euler and Navier-Stokes equations and the second law of Thermodynamics. Computer Methods in Applied Mechanics Engineering, Amsterdam, v.54, n.2, p.223-234, feb. 1986.

LEDERER, F.; BERTOLOTTI, M.; SIBILIA, C.; LEUTHEUSER, E. V. An externally controlled nonlinear directional coupler. Optics Communications, Amsterdam, v.75, n.3/4, p.246250, mar. 1990.

MALOMED, B. A.; MIHALACHE, D.; WISE, F.; TORNER, L. Spatiotemporal optical solitons. Journal of Optics B, Bristol, v.7, n.5, p.R53-R72, may 2005.

MENYUK, C. R.; SCHIEK, R.; TORNER, L. Solitary waves due to $\chi^{(2)}$ : cascading. Journal of the Optical Society of America B - Optical Physics, Washington, v.11, v.12, p.2434-2443, dec. 1994.

QIN, Y.; DAI, C. Q.; ZHANG, J. F. Higher-order effects induced optical solitons in fiber. International Journal of Theoretical Physics, New York, v.44, n.8, p.1117-1121, aug. 2005.

RAGHAVAN, S.; AGRAWAL, G. P. Spatiotemporal solitons in inhomogeneous nonlinear media. Optics Communications, Amsterdam, v.180, n.4/6, p.377382, jun. 2000.
ROMEIRO, N. M. L.; CASTRO, R. G. S; LANDAU, L. Simulação numérica de modelos de qualidade de água usando o método de elementos finitos estabilizados. Tese (Doutorado) Universidade Federal do Rio de Janeiro, Rio de Janeiro.

STROBEL, O. A. Fiber-optic transmission - an overview. Telecomunicações, Santa Rita do Sapucaí, v.7, n.2, p.1-9, dez. 2004.

TAYLOR, J. R. Optical solitons - Theory and experiments. Cambridge: Cambridge University Press, 1992.

WERNER, M. J.; DRUMMOND, P. D. Simulton solutions for the parametric amplifier. Journal of the Optical Society of America B - Optical Physics, Washington, v.10, n.12, p.2390-2393, dec. 1993.

YMAI, L. H.; GALLEAS, W.; NATTI, P. L.; TAKANO NATTI, E. R. Estabilidade de sólitons em fibras óticas dielétricas de tipo . Revista Ciências Exatas e Naturais, Guarapuava, v.6, p.9-30, 2004.

ZAKHAROV, V. E.; SABAT, A. B. Exact theory of 2-dimensional self-focusing and one-dimensional self-modulation of waves in nonlinear media. Soviet Physics JETP-USSR, New York, v.34, n.1, p.62-69, 1972. 